\title{
PIV measurements of induced separation at the leading edge of a plate
}

\author{
J. P. J. Stevenson ${ }^{1} \dagger$, K. P. Nolan ${ }^{2}$ and E. J. Walsh ${ }^{3}$ \\ ${ }^{1}$ Stokes Laboratories, University of Limerick, Limerick, Ireland \\ ${ }^{2}$ Thermal Management Research Group, Bell Labs Ireland, Alcatel-Lucent, Dublin, Ireland \\ ${ }^{3}$ Department of Engineering Science, University of Oxford, Oxford, UK
}

(Received $\mathrm{xx}$; revised $\mathrm{xx}$; accepted $\mathrm{xx}$ )

The free shear layer that separates from the leading edge of a round-nosed plate has been studied under conditions of low (background) and elevated (grid-generated) freestream turbulence (FST) using high-fidelity PIV. Transition occurs after separation in each case, followed by reattachment to the flat surface of the plate downstream. A bubble of reverse flow is thereby formed. First, we find that under elevated (7\%) FST, the timemean bubble is almost threefold shorter due to an accelerated transition of the shear layer. Quadrant analysis of the Reynolds stresses reveals the presence of slender, highly coherent, fluctuations amid the laminar part of the shear layer that are reminiscent of the boundary-layer streaks seen in bypass transition. Instability and the roll-up of vortices then follow near the crest of the shear layer. These vortices are also present under low FST and in both cases are found to make significant contributions to the production of Reynolds stress over the rear of the bubble. But their role in reattachment, whilst important, is not yet fully clear. Instantaneous flow fields from the low-FST case reveal that the bubble of reverse flow often breaks up into two or more parts, thereby complicating the overall reattachment process. We therefore suggest that the downstream end of the 'separation isoline' (the locus of zero absolute streamwise velocity that extends unbroken from the leading edge) be used to define the instantaneous reattachment point. A histogram of this point is found to be bimodal: the upstream peak coincides with the location of roll-up, whereas the downstream mode may suggest a 'flapping' motion.

\section{Key words:}

\section{Introduction}

Turbulent boundary layers that follow regions of separation are incredibly slow to reach equilibrium (Castro \& Epik 1998). Yet boundary layers of this type are commonly found in practice, and so an understanding of the conditions that produce them is crucial. In this work we study the case of a round-nosed, smooth flat plate. A trailing-edge flap is used to induce separation at the leading edge and generate a transitional shear flow that would provide the initial conditions for a reattaching turbulent boundary layer downstream. The Reynolds number $\operatorname{Re}_{D}$, based on the diameter $D$ of the fine semi-cylindrical leading edge, is about 800 , which is much lower than the majority of studies on this class of flow. Also considered are the effects of free-stream turbulence (FST). As discussed by

$\dagger$ Email address for correspondence: jonathan.stevenson@ul.ie 
Yang \& Abdalla (2008), experimental studies on the large-scale structures that develop in separated shear flows under FST are rather limited, and so it is also our intention here to provide some new physical insight on the mechanisms involved.

In this survey, we focus mainly upon those studies resembling our own i.e. those that address separating flow at the leading edge of blunt or round-nosed flat plates at relatively low Reynolds number. The thickness of the (laminar) shear layer in these cases is practically zero at detachment, which importantly distinguishes them from those that arise by separation of relatively thick, and possibly turbulent, boundary layers with an upstream history e.g. laminar separation bubbles (LSBs) on aerofoils at high incidence (see Tani 1964). First of all, it is important to establish what is meant by 'relatively low' in this context because, in general, it is the Reynolds number that governs the scale and dynamics of flows with laminar separation (Li \& Djilali 1995).

\subsection{Reynolds-number regimes in separation-reattachment flow}

Ota et al. (1981) investigated the effect of Reynolds number $\operatorname{Re}_{t}=U_{0} t / \nu$ on the mean length $l_{\mathrm{R}}$ of the leading-edge separation bubble over a flat plate, of thickness $t$, in a water channel. For each of the tested leading edges, which included blunt, semi-cylindrical and various pointed designs, a consistent pattern of behaviour was observed: (i) at low Reynolds numbers, the shear layer above the bubble would both separate and reattach in laminar fashion, with attached transition following downstream; (ii) at low-moderate Reynolds numbers, transition of the shear layer would occur mid-separation; and (iii) at high Reynolds numbers, the layer would both separate and reattach in turbulent fashion, transition having started almost instantaneously after detachment. For the semicylindrical leading edge, $l_{\mathrm{R}}$ increased sharply in regime (i) until about $\mathrm{Re}_{t}=600$, peaked at nearly $4 t$, and then decreased slowly in regimes (ii) and (iii). The data indicated that at sufficiently high Reynolds numbers the bubble might even vanish entirely (in contrast, the experiments of Lane \& Loehrke (1980) on a sharp-edged blunt plate show that $l_{\mathrm{R}}$ reaches a distinct limit of $4.5 t$ at around $\operatorname{Re}_{t}=600$ ). Much of the detailed work on separation-reattachment over plates has been carried out at Reynolds numbers far into (iii), with the intention that the bubble zone be fully turbulent e.g. Cherry et al. (1984). In this work, though, the Reynolds number was low enough for laminar flow to comprise a significant part of the shear layer in both cases i.e. the flow belongs in regime (ii).

Sasaki \& Kiya (1991) investigated the nature of the breakdown process over a blunt plate in (ii) and at the low end of (iii) by tracking the development of vortex lines within the separated shear layer. They first observed a gentle unsteadiness at $\operatorname{Re}_{t} \simeq 320$ in the form of a travelling wave, which propagated downstream without causing any spanwise distortion of the flow. But as $\mathrm{Re}_{t}$ was increased beyond about 325, the shear layer started to roll up via Kelvin-Helmholtz $(\mathrm{KH})$ instability into an almost two-dimensional vortex sheet along the width of the plate. The corresponding vortex line (at the core of the sheet) subsequently assumed a periodic, wave-like character around reattachment, before dividing itself into a repeating array of $\Lambda$-type structures in the spanwise direction. Breakdown did not proceed until after reattachment (outside their field of view). Studies in regime (iii) show that these $\mathrm{KH}$ instabilities continue at high turbulent Reynolds numbers (Kiya \& Sasaki 1985; Yang \& Voke 2001; Lamballais et al. 2010), with the point of their onset moving upstream in accordance with the Reynolds number. The rolled-up vortices also tend to amalgamate with one another-see Hwang et al. (2000) for a detailed visualisation of this - to form large, staggered, three-dimensional hairpin structures, and consequently the characteristic time scale of the flow increases in the downstream direction until reattachment (Kiya \& Sasaki 1983), where it plateaus. The 
pseudo-periodic shedding frequency usually reported in turbulent separation bubbles $\left(\simeq 0.6 U_{0} / l_{\mathrm{R}}\right)$ is primarily associated with the passage of these large-scale structures.

\subsection{Low-frequency unsteadiness}

Eaton \& Johnston (1982) suggested from their backstep experiments that separated shear layers might also undergo non-periodic vertical oscillations - since termed 'flapping' by some - that cause the instantaneous reattachment point to drift back and forth on a relatively long time-scale. They proposed that the motion might result from an instantaneous imbalance between reinjection near reattachment and entrainment upstream, although the underlying mechanics were still unclear. Interestingly, the flapping issue continues to divide the community e.g. see Castro \& Haque (1987), Djilali \& Gartshore (1991) and Kiya \& Sasaki (1983) for studies with strong evidence, and Chandrsuda \& Bradshaw (1981), Ruderich \& Fernholz (1986) and Yang \& Abdalla (2009) otherwise. Now whilst it may well be in the interpretation of what constitutes 'flapping' that their differences lie, common to the studies - most of which concern turbulent flow - that do report such a motion is the idea that the bubble bounded by the shear layer undergoes broad expansion-contraction cycles, as originally described by Eaton \& Johnston (1982). The motion is certainly not geometry-specific and takes place at a much lower frequency than 'regular' large-scale shedding $(\S 1.1)$.

Hudy et al. (2003) suggested that flapping might be the response of the shear layer to a zone of absolute instability within the underlying bubble of reverse flow. The global mode described by Castro (2005) furnishes a physical description for the resultant motion, namely, the periodic shedding of 'lumps' of vorticity from the rear of the bubble. When vorticity is shed, the first stagnation or reattachment point suddenly jumps upstream, only to drift gradually downstream again when the cycle repeats itself. Both Hudy et al. (2003) and Castro (2005) point out that the mere existence of a reverse-flow region is not a sufficient condition for the global mode; the backflow velocity must be large, probably in excess of $0.2 U_{0}$, and the Reynolds number clearly above that for absolute instability.

\subsection{The effects of FST}

FST tends to promote all aspects of development in separated flows, including spreading, mixing, entrainment and the transition to turbulence (Bearman \& Morel 1983). Kiya \& Sasaki (1985) also report an increase in the integral time-scale near separation points. All else being equal, each mechanism usually serves to reduce the size of a reverse-flow region (Hillier \& Cherry 1981; Castro \& Haque 1988; Kalter \& Fernholz 2001).

There are relatively few studies, particularly experimental, that address the transition process in free shear layers over plates under elevated FST, particularly at low Reynolds numbers. Yang \& Abdalla (2005) investigated the effect of $2 \%$ FST on separated transition over a blunt plate at $\mathrm{Re}_{t}=6500$ using LES. Immediately after detachment, instability resembled the $\mathrm{KH}$ mechanism that prevails in the clean case, whereas downstream the observed structures were not readily identifiable and the path to turbulence was supposed to be different. Under higher (5.6\%) FST, the subsequent simulations of Langari \& Yang (2013) at $\operatorname{Re}_{t}=3450$, for the case of the semi-cylindrical nose, showed a very rapid transition-to-turbulence in which $\mathrm{KH}$ instability seemed to disappear altogether. They suggested the existence of a 'bypass' route in analogy to the case of attached boundary-layer transition. To our knowledge, theirs is the only work to describe separated transition under elevated FST in these terms.

First, in $\S 2$, the experiment setup is described. The flap effect is then demonstrated in $\S 3$, followed by a detailed discussion of the time-mean and instantaneous flow fields in 


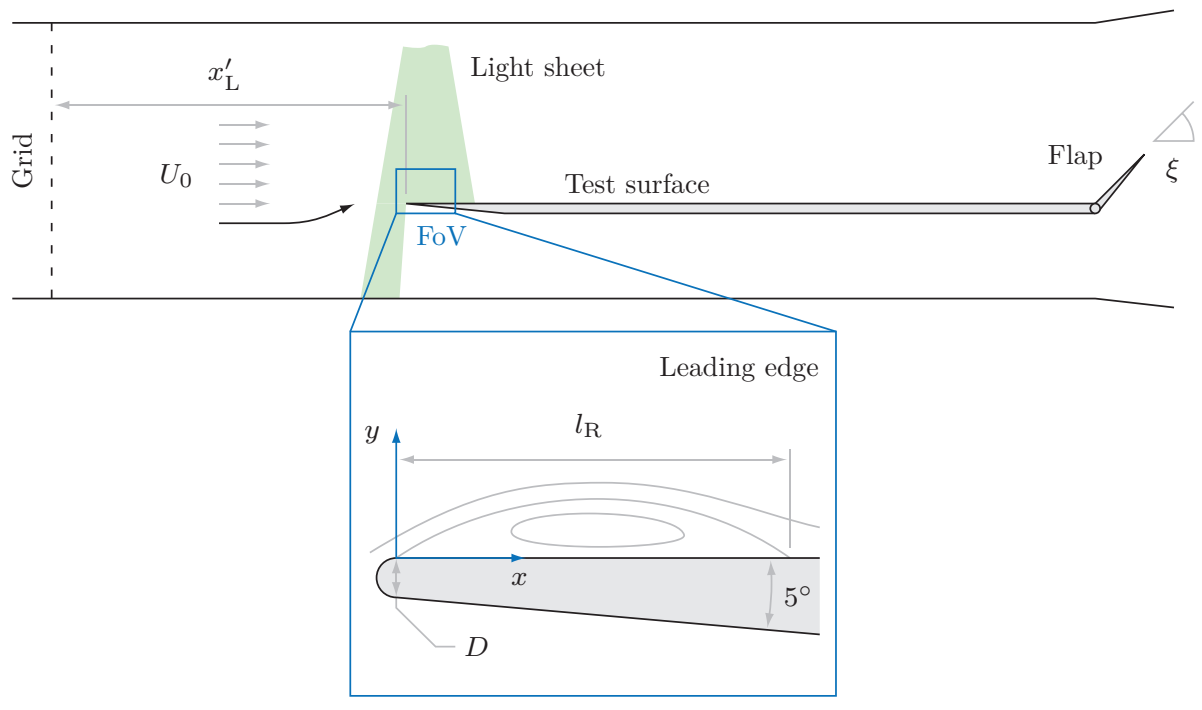

Figure 1: Cross-sectional view of the working section, grid, test plate and PIV setup (not to scale). The zoom-view shows the geometry of the leading edge.

each case. The cases are denoted case A (background FST) and case B (elevated FST) for reference. Finally, conclusions are drawn in $\S 4$.

\section{Experiment setup}

\subsection{Facility \& PIV instrumentation}

All measurements were carried out in an open-return, suckdown wind tunnel over a flat aluminium plate (figure 1). The tunnel consists of a bell-mouthed inlet, settling chamber (with a coarse honeycomb and a fine woven-mesh screen), contraction, working section $(300 \mathrm{~mm} \times 300 \mathrm{~mm} \times 1000 \mathrm{~mm})$, diffuser (with two screens) and four axial fans in parallel. The background turbulence level $T_{u \mathrm{~b}}$ in the empty working section is $0.45 \%$.

The plate is $295 \mathrm{~mm}$ wide and is made up of three sections: (i) an asymmetrical leading edge, the underside of which is tapered at $5^{\circ}$ from the plate proper $(10 \mathrm{~mm}$ thick) to a semi-cylindrical nose of diameter $D=2 \mathrm{~mm}$; (ii) a long test surface, which is smoothly conjoined to the leading edge; and (iii) a hinged trailing-edge flap (85 mm long), which allows the incidence of the approaching free-stream to be manipulated indirectly. When the flap is set to a low angle $\xi$, a separation bubble of time-mean length $l_{\mathrm{R}}$ will appear on the test surface. The full plate is $920 \mathrm{~mm}$ long, excluding the flap.

The PIV system (from TSI Inc.) comprised a $200 \mathrm{~mJ}$ pulsed Nd:YAG laser, an $11 \mathrm{MP}$ CCD camera (with a Sigma f/2.8 $105 \mathrm{~mm}$ prime lens) and a commercial fog machine. The field-of-view (FoV) in the laser light-sheet $(x-y)$ plane was $42 \mathrm{~mm} \times 28 \mathrm{~mm}$ and was offset by $10 \mathrm{~mm}$ from the centreline of the plate to accommodate the focus distance to the lens. In each case, 1000 image pairs were captured at a frequency of 0.55 pairs per second, as limited by the write speed of the storage drive. The vector field was later computed recursively across a rectangular grid of interrogation spots (down to a spacing of $93 \mu \mathrm{m}$ per vector) using the second-order deformation algorithm in TSI Insight. This algorithm is based upon the iterative multigrid method of Scarano (2002) and is 
particularly effective against in-plane velocity gradients. Each field was also corrected for mild pincushion distortion from the lens, although the effect was negligible.

Total uncertainty in PIV velocity measurements depends upon both bias (due to equipment, calibration, etc.) and random errors (which vary across the flow field according to local image quality, fluid strain and rotation, particle-image characteristics, etc.). The former being known, the latter were estimated using the Insight implementation of the peak ratio method (Charonko \& Vlachos 2013). In case A, for example, the total standard uncertainty in the unsteady region to the rear of the bubble was typically 5-10\%, whereas in the laminar shear layer (and external stream), it was 1-3\%. Amid the upstream part of the bubble, it rose to $20-25 \%$, but here we were concerned only with the streamwise direction of the flow (to deduce the reattachment point) and not its magnitude.

\subsection{Free-stream turbulence}

For case B, free-stream turbulence (FST) was generated upstream of the plate by means of a $2 \mathrm{~mm}$-thick perforated grid. The bars of the grid are $d=7 \mathrm{~mm}$ wide and spaced evenly at a mesh length of $M=26 \mathrm{~mm}$. The turbulence was characterised in the empty working section of the tunnel with an AN-1005 hot-wire anemometer (from AA Lab Systems) and a 55P11 single-wire probe (from Dantec) that had been calibrated in situ beforehand against a Pitot-static tube using King's law. Fluctuations in ambient temperature were corrected by the method of Kanevče \& Oka (1973).

The decay of streamwise turbulence intensity $T_{u}$ with dimensionless distance $x^{\prime} / d$ behind the grid is shown in figure 2 (a). For comparison, the data are plotted against the power-law correlation from Roach (1987),

$$
T_{u}=\frac{\sqrt{\overline{u^{\prime 2}}}}{U_{0}}=A\left(\frac{x^{\prime}}{d}\right)^{-\frac{5}{7}},
$$

with $A=1.13$ (Roach's value for this grid type and Reynolds number). $\sqrt{\overline{u^{\prime 2}}}$ is the rootmean-square level of the streamwise fluctuations $u^{\prime}$, where the overline denotes the timeaveraging operation. After an initial settling region, the measured turbulence decays at approximately the same rate as Roach's line. The leading edge of the plate was therefore positioned at the end of this region $\left(x_{\mathrm{L}}^{\prime}=331 \mathrm{~mm}\right.$ or $\left.x_{\mathrm{L}}^{\prime} / d=47.3\right)$ in order to avoid any development effects near the grid. Figure 2 (b) shows the streamwise correlation function at this location, computed as an autocorrelation of the $u^{\prime}$ signal or

$$
R_{u u}=\frac{\overline{u^{\prime}(t) u^{\prime}(t+\tau)}}{\overline{u^{\prime 2}}},
$$

and this matches Dryden's function (Dryden 1943) for isotropic turbulence,

$$
R_{u u}=\exp \left(-\frac{U_{0} \tau}{\Lambda_{u}}\right),
$$

quite closely. Note that $R_{u u}$ has been plotted against the dimensionless quantity $U_{0} \tau / \Lambda_{u}$, where $\tau$ is the time-lag variable (product $U_{0} \tau$ is therefore equivalent to the streamwise separation distance in a two-point correlation) and $\Lambda_{u}$ is the streamwise integral lengthscale. $\Lambda_{u}$ was estimated by integration of $R_{u u}$ with respect to $\tau$ as

$$
\Lambda_{u}=U \int_{0}^{\tau_{\mathrm{L}}} R_{u u}(\tau) \mathrm{d} \tau .
$$

Upper limit $\tau_{\mathrm{L}}$ was defined as the first point at which $R_{u u}=0.02$ to prevent any 'noisy' 

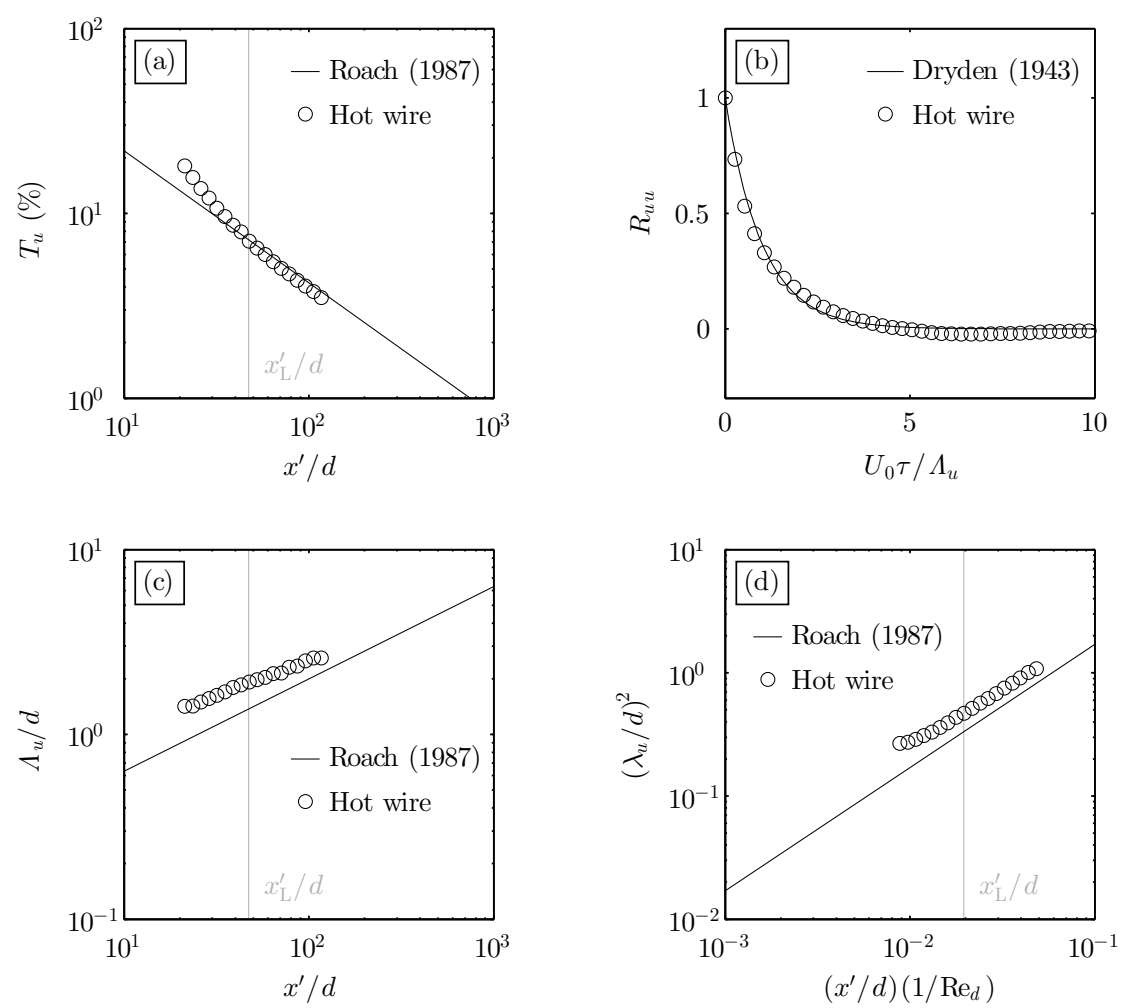

Figure 2: Characteristics of the grid-generated FST. Plots (a), (c) and (d) show the development of the streamwise turbulence intensity $T_{u}$, integral length-scale $\Lambda_{u}$ and Taylor microscale $\lambda_{u}$, respectively, with distance $x^{\prime} / d$ behind the grid. In (b), which shows the correlation function $R_{u u}$ at the location of the leading edge $\left(x_{\mathrm{L}}^{\prime} / d=47.3\right)$, only every $25^{\text {th }}$ data point is shown for clarity (the original data are very highly resolved).

oscillations that might appear at large $\tau$ from affecting the integral sum. This follows from the study of O'Neill et al. (2004), who used the first zero-crossing point of $R_{u u}$.

The streamwise growth of $\Lambda_{u}$ is shown in figure 2 (c). Roach's (1987) universal correlation for all grid types,

$$
\frac{\Lambda_{u}}{d}=0.2\left(\frac{x^{\prime}}{d}\right)^{\frac{1}{2}},
$$

is also shown. We note that his choice of growth exponent (0.5) was a general assumption, made for reasons given in his paper, and that the leading coefficient (0.2) was determined by regression to a multitude of data sets. Whilst the agreement is therefore reasonable given the generality of the correlation, we find from an optimised curve-fit to our data that the present exponent is actually 0.37 , and the leading coefficient 0.45 . This is more consistent with the data of Kurian \& Fransson (2009), who find an exponent of 0.33 . Nonetheless, the correlation provides the requisite benchmark for comparison.

The Taylor microscale $\lambda_{u}$ provides a measure of the smallest energetic eddies in the 
flow i.e. anything smaller falls into the viscous dissipation range. It is defined by

$$
\frac{1}{\lambda_{u}^{2}}=-\frac{1}{2 U_{0}^{2}}\left[\frac{\partial^{2} R_{u u}}{\partial \tau^{2}}\right]_{\tau=0} .
$$

In this work, $R_{u u}$ was sufficiently well resolved at small $\tau$ for $\lambda_{u}$ to be approximated using a simple finite difference. The computed results are plotted in figure 2 (d) alongside Roach's (1987) corresponding correlation,

$$
\left(\frac{\lambda_{u}}{d}\right)^{2}=17\left(\frac{x^{\prime}}{d}\right)\left(\frac{1}{\operatorname{Re}_{d}}\right),
$$

which may be obtained from theory and equation 2.1. Note that $\operatorname{Re}_{d}=U_{0} d / \nu$ is the bar Reynolds number of the grid. Despite a small vertical offset, the growth exponent here compares favourably to the data after the initial settling region.

These characteristics suggest overall that the turbulence is well behaved and, on the basis of 2 (b) in particular, sufficiently isotropic for this work. PIV data from case B also show that the mode of the ratio $\overline{u^{\prime 2}} / \overline{v^{\prime 2}}$ in the free-stream is approximately 1.04 .

\section{Results \& discussion}

Both separated flows were generated by means of the hinged flap at the trailing edge of the plate (figure 1). Before a detailed discussion of the flow, a brief description of the 'flap effect' therefore follows.

\subsection{The flap effect}

For attached boundary-layer experiments on plates, it is imperative that separation is prevented at the leading edge. One common method is to use an asymmetrical leadingedge section in conjunction with a raised trailing-edge flap: the leading edge provides a smooth blend from the plate proper (usually quite thick for structural reasons) to a finely rounded nose, and the flap provides the circulation necessary for the flow to attack the plate at negative incidence. This ensures that the stagnation point remains firmly anchored to the nose (Hernon et al. 2007) so that separation is avoided. The attendant boundary layer might require a virtual origin for proper scaling, but under constantpressure conditions will usually follow Blasius' solution after a short initial development length at the leading edge.

The present plate was designed in accordance with similar guidelines from Roach \& Brierley (1992). Attached flow is obtained when the elevation angle $\xi$ of the flap reaches approximately $45^{\circ}$; in this configuration the boundary layer that grows downstream is laminar and matches Blasius' scaling well (figure 3). But as the flap is lowered from $\xi=45^{\circ}$, the circulation strength decreases and the approach angle of the incident flow increases. Eventually the flow can no longer remain attached and separates from the nose to form a bubble of reversing flow. The effect is demonstrated in figure 4 (reproduced from Stevenson et al. 2014) for three flap settings and FST intensities, albeit at a slightly lower Reynolds number than the present work.

The flap setting of $\xi=0^{\circ}$ was chosen for further study at two levels of FST. Details of these experiments, including the turbulence characteristics at the leading edge, are provided in table 1, and contours of mean velocity are presented in figure 5. As described previously, the action of the flap (or lack thereof) causes the incident flow to approach at an elevated angle such that separation is forced at the leading edge. A thin band of shear, really a free shear layer, subsequently develops between the separated, highvelocity fluid in the free-stream and the underlying low-velocity region, which at this 

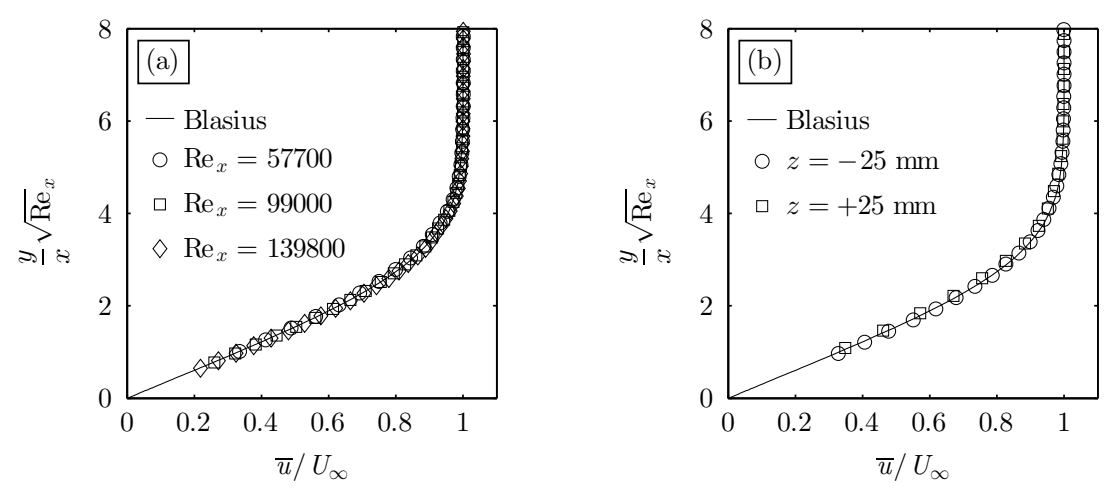

Figure 3: Velocity profiles of the attached, laminar boundary layer that grows on the test surface when $\xi=45^{\circ}$ and the external velocity $U_{\infty} \simeq 4.9 \mathrm{~m} \mathrm{~s}^{-1}$. Plot (a) shows growth along the centreline, and plot (b) the profiles $25 \mathrm{~mm}$ on either side of this line (in the cross-stream or $z$ direction) at $\operatorname{Re}_{x}=57700$ in order to demonstrate spanwise homogeneity. The shape factor $H_{12}$ for these profiles is in the range 2.53-2.60, confirming that the streamwise pressure gradient is small (Blasius' profile yields $H_{12}=2.59$ ). Note that a virtual origin of $+10 \mathrm{~mm}$, a downstream shift, has been applied; $x$ therefore extends from the new coordinate origin.
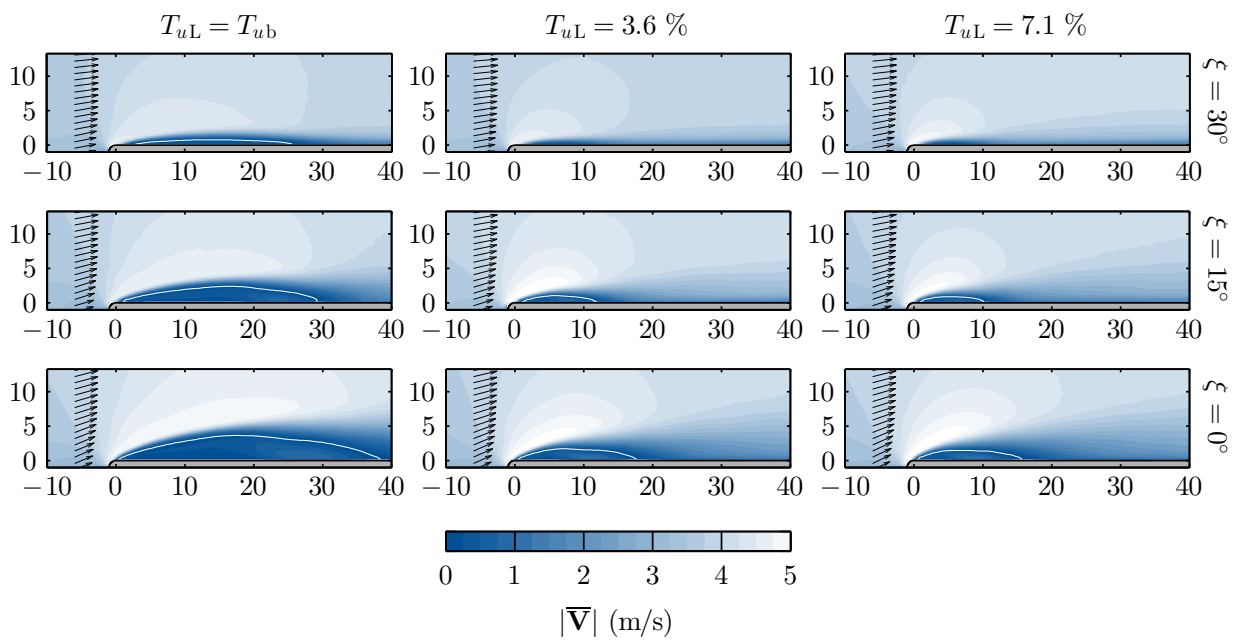

Figure 4: Contours of the mean absolute velocity magnitude $|\overline{\mathbf{V}}|$ at the leading edge for 9 separated flows (from Stevenson et al. 2014). $\operatorname{Re}_{D}=480 \pm 20$. Isolines of $\bar{u}=0$ are shown by white lines. The profile of incident vectors at $x=-6 \mathrm{~mm}$ has also been included in each case. Axes units are $[\mathrm{mm}]$.

Reynolds number is strong enough to initiate roll-up and ultimately the transition to turbulence. The flow then reattaches to form a recirculating separation bubble that is driven continuously by the shear. Under elevated FST (case B), the bubble shortens almost threefold from $24.4 \mathrm{~mm}$ to $8.9 \mathrm{~mm}$. Its centre of 'rotation' (in terms of $l_{\mathrm{R}}$ ) is also brought forward, whilst the maximum backflow velocity remains approximately the 


$\begin{array}{lcccccc}\text { Case } & U_{0}\left(\mathrm{~m} \mathrm{~s}^{-1}\right) & \operatorname{Re}_{D} & T_{u \mathrm{~L}}(\%) & \Lambda_{u \mathrm{~L}}(\mathrm{~mm}) & \lambda_{u \mathrm{~L}}(\mathrm{~mm}) & l_{\mathrm{R}}(\mathrm{mm}) \\ \text { A } & 5.9 & 790 & T_{u \mathrm{~b}} & - & - & 24.4 \\ \text { B } & 5.9 & 790 & 7.1 & 13.5 & 8.2 & 8.9\end{array}$

Table 1: Experiment parameters in each case.

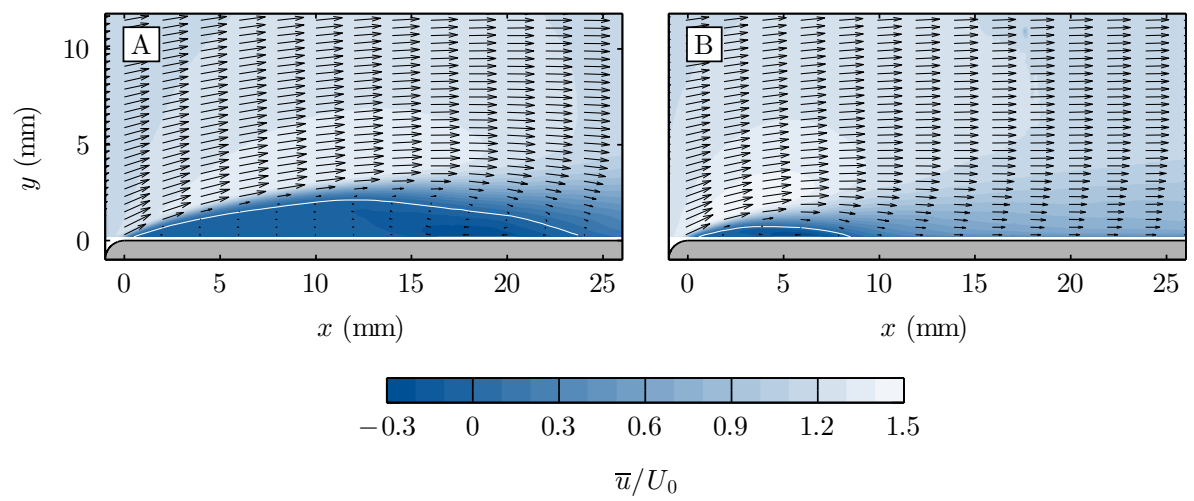

Figure 5: Contours of the streamwise velocity $\bar{u} / U_{0}$ in each case. Isolines of $\bar{u} / U_{0}=0$ are shown by white lines. Coarse vector profiles of the mean flow have also been overlaid.

same at $\bar{u} / U_{0} \simeq-0.3$ (the importance of this is discussed in $\S 4$ ). That FST tends to shrink or even eliminate separation bubbles has been observed by several workers at high Reynolds numbers (e.g. Kalter \& Fernholz 2001) and is usually attributed to the effects of enhanced entrainment. At the present low Reynolds number, the shrinkage effect results primarily from an accelerated transition of the shear layer (§3.2).

\subsection{Fluctuations in the shear layer}

Perhaps the clearest overall perspective of the bubble dynamics is provided by the field of instantaneous spanwise vorticity (figure 6). In each case, the development of the flow is observed to consist of four essential parts: (i) the establishment and growth of the free shear layer from the point of separation, as shown by the smooth band of (diminishing) negative vorticity that extends from the leading edge; (ii) the onset of roll-up near the crest of the shear layer, which usually manifests as a circular patch of nearly constant negative vorticity (actually vortex roll-up, as later shown in the flow fields of figures 10 and 11); (iii) breakdown of the shear layer to turbulence, during which a wide range three-dimensional motions - revealed by the divergence field, though not shown - start to appear, and finally; (iv) reattachment to the wall to form a new turbulent boundary layer. Further, the right-hand plot shows that the point of roll-up in the shear layer is significantly advanced in case B. This mechanism, in combination with the enhanced spreading and mixing that take place naturally under elevated FST (Bearman \& Morel 1983), serve to bring forward the first point of reattachment, and this in turn accounts for the observed reduction in the length of the bubble.

In a time-mean sense, the unsteadiness associated with the transition process is well 

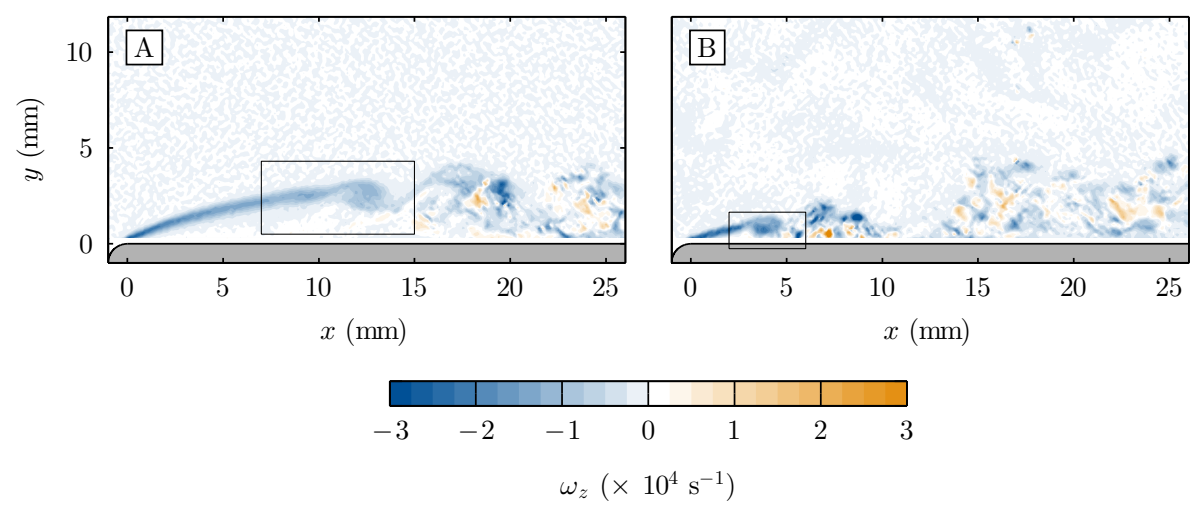

Figure 6: Contours of the spanwise vorticity component $\omega_{z}$ for an instantaneous flow field in each case. The black windows are referenced in figures 10 and 11 .

described by the two-component turbulence intensity $T_{u v}$ (figure 7 ), defined as

$$
T_{u v}=\frac{\sqrt{\frac{1}{2}\left(\overline{u^{\prime 2}}+\overline{v^{\prime 2}}\right)}}{U_{0}} .
$$

$u^{\prime}=u-\bar{u}$ and $v^{\prime}=v-\bar{v}$ are the Reynolds-decomposed velocity fluctuation components. Sasaki \& Kiya (1985) also suggest that the locus of maximum turbulence intensity may be used to identify the centreline, and hence trajectory, of a separated shear layer, and so this has been added to each contour in figure 7 . We note that laminar portion of the shear layer is reasonably steady in each case, and consequently the time-mean and instantaneous (figure 6) paths match one another quite well. An accompanying line plot of $\left(T_{u v}\right)_{\max }$ has also been included at the top of the figure. This shows the familiar 'growth-peak-decay' behaviour that is fundamental to the transition process; Fransson et al. (2005) present a similar plot for the case of attached bypass transition, as do Langari \& Yang (2013) for their separated flow. In case B, initial events are clearly accelerated, in that $\left(T_{u v}\right)_{\max }$ grows more rapidly and reaches its peak much earlier, but downstream the decay rates turn out to be very similar. This has been illustrated by re-plotting the data for case B at a distance of $14 \mathrm{~mm}$ downstream but in light-coloured solid circles. Note that the shift is comparable to the difference between the time-mean bubble lengths $(=15.5 \mathrm{~mm})$. The similarity could suggest that some form of 'shear-sheltering' effect, provided by efflux from the bubble - see Hunt \& Durbin (1999) for the original idea and Kalter \& Fernholz (2001) in this context - shields the reattaching boundary layer, so that after a certain point its development no longer depends upon the external turbulence. But whilst an interesting possibility, certainly from the perspective of boundary-layer control, one cannot draw firm conclusions without further data in the downstream direction.

We have found the method of Reynolds-stress decomposition, often known simply as quadrant analysis, to be particularly effective in describing the physical nature of the fluctuations within the shear layer. The idea was first introduced by Lumley (1970) and is useful in almost all fluctuating shear flows for identifying the main contributors to momentum transfer. For discrete data, the total Reynolds stress $\overline{u^{\prime} v^{\prime}}$ is usually calculated as the time average of contributions from the instantaneous fluctuation product $u^{\prime} v^{\prime}$, which can either be positive or negative depending on the individual signs of $u^{\prime}$ and $v^{\prime}$. If $u^{\prime} v^{\prime}>0$, then either $u^{\prime}$ and $v^{\prime}$ are both positive or both negative; the product 

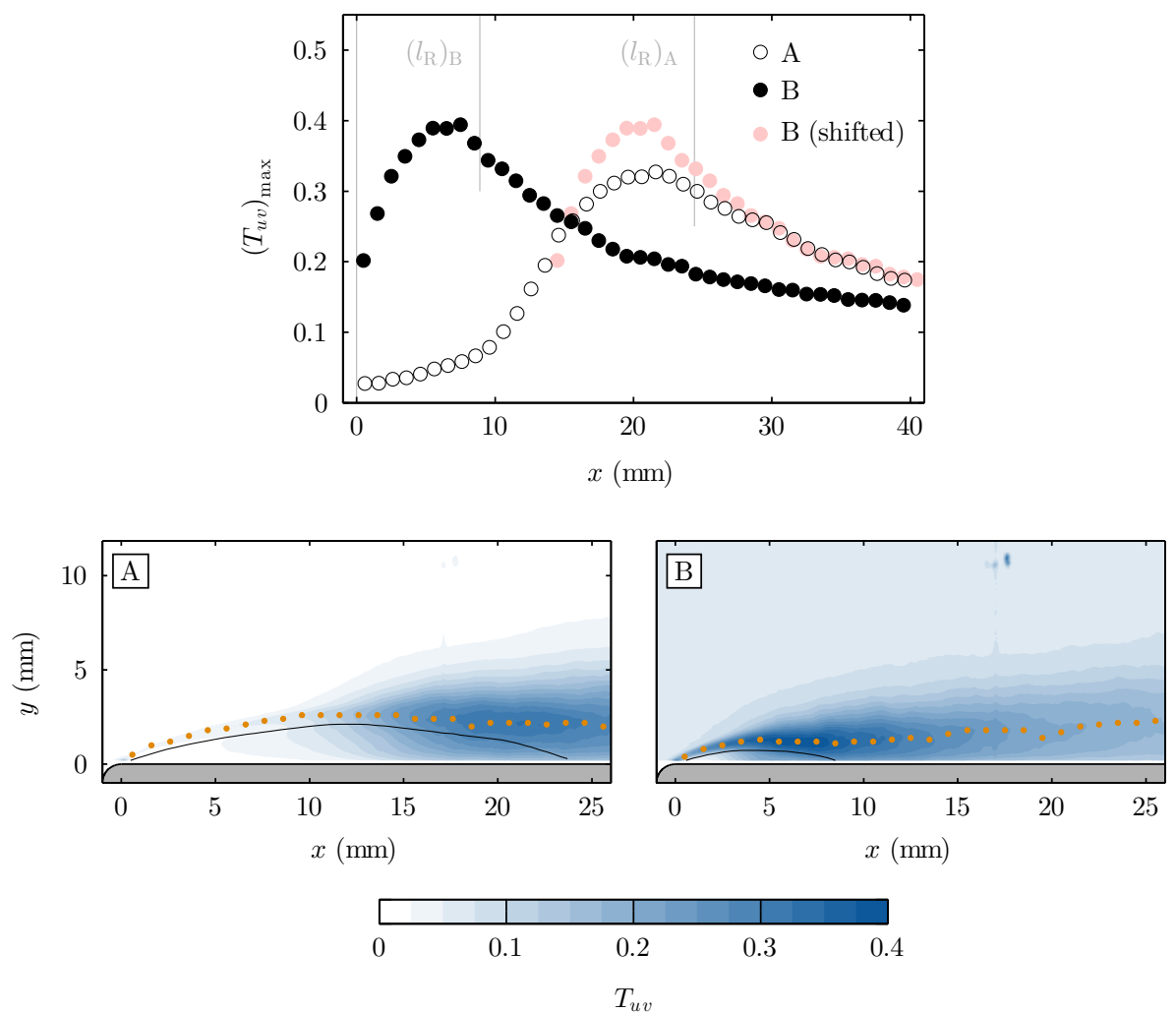

Figure 7: Contours of $T_{u v}$ in each case, with orange dots marking the loci of $\left(T_{u v}\right)_{\max }$ at every $10^{\text {th }}$ streamwise grid point. Isolines of $\bar{u} / U_{0}=0$ are shown in black. A corresponding line plot of $\left(T_{u v}\right)_{\max }$ along each locus has been included at the top of the figure. Note that the abscissa limits on the line plot extend to the extreme right-hand edge of the PIV field of view (not required by other plots) to show the decay phase.

therefore belongs in quadrant 1 or 3 . If $u^{\prime} v^{\prime}<0$, then the product belongs in quadrant 2 or 4. 'Quadrant' (denoted Q) here has its usual meaning: one of the four sectors in the Cartesian $u^{\prime}-v^{\prime}$ plane. At each point in the flow, the total stress $\overline{u^{\prime} v^{\prime}}$ can therefore be decomposed into contributions $\widetilde{u^{\prime} v^{\prime}}{ }_{i}$ from each quadrant $i$ as

$$
\overline{u^{\prime} v^{\prime}}=\sum_{i=1}^{4} \widetilde{u^{\prime} v^{\prime}}{ }_{i}
$$

where

$$
\widetilde{u^{\prime} v^{\prime}}{ }_{i}=\frac{1}{N} \sum_{j=1}^{N_{i}}\left(u^{\prime} v_{i}^{\prime}\right)_{j} .
$$

$N$ is the total number of measurement samples, and $N_{i}$ the number that lie in quadrant $i$ i.e. $N=\sum N_{i}$. Now in the case of the boundary layer on a flat surface, whose time-mean streamlines are virtually parallel, quadrant analysis at each point is usually carried out in the fixed wall coordinate system ( $x-y$ in this work) so that the sense of the fluctuations is clear. For curved shear layers like the present, however, it is perhaps more appropriate 

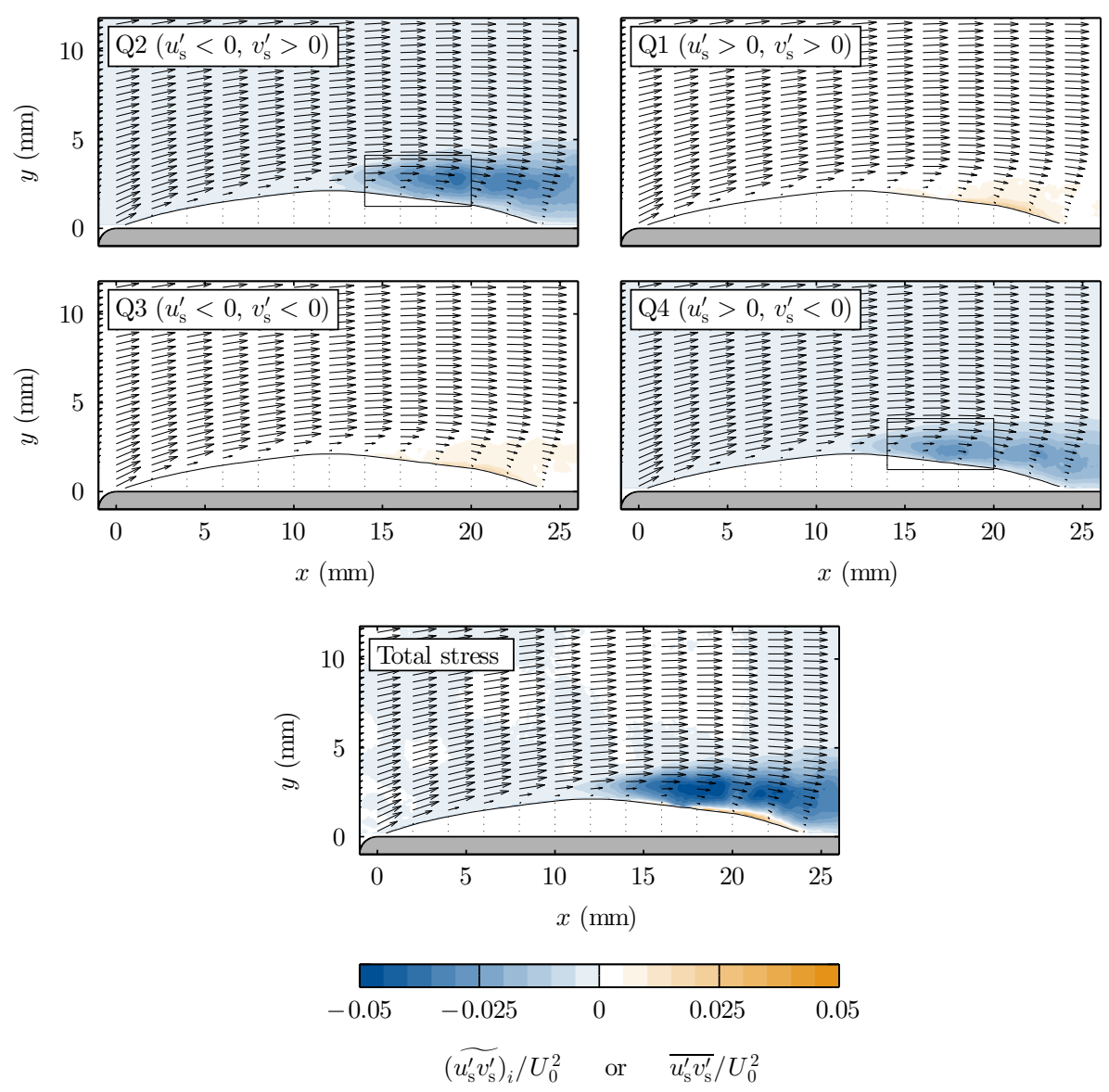

Figure 8: Contours of the stress contribution $\widetilde{\left(u_{\mathrm{s}}^{\prime} v_{\mathrm{s}}^{\prime}\right)_{i}} / U_{0}^{2}$ from each quadrant in case A (excluding the bubble region). Total Reynolds stress $\overline{u_{\mathrm{s}}^{\prime} v_{\mathrm{s}}^{\prime}} / U_{0}^{2}$ is shown at the base of the figure. Isolines of $\bar{u} / U_{0}=0$ are shown in black. Coarse profiles of the corresponding mean vector field have also been overlaid.

to work in a local system aligned with the mean streamlines, the main requirement being that the flow display a clear direction of development (Castro \& Haque 1987). We have therefore decided to adopt this approach. The velocity fluctuations in this system have been distinguished by a subscript 's' for 'streamline', and in any subsequent reference to a 'Reynolds stress' the use of this system is implied. Note that the bubble of reverse flow has been omitted; the flow direction here is not amenable to the analysis.

Figures 8 and 9 show contours of the quadrant-decomposed Reynolds stress around the bubble in cases A and B, respectively. All stresses are practically zero in the laminar part of the shear layer; those in Q1 and Q3 remain that way for most of the bubble. However, there is a thin band of orange downstream that - if one envisages the orientation of a set of 'quadrant axes' in this region-corresponds, for Q1 and Q3 respectively, to the recirculation of relatively low-speed (reversing) fluid back into the bubble and the deflection of relatively high-speed fluid into the new wall layer. This is one of the key processes of reattachment.

At $x \simeq 12 \mathrm{~mm}$ in case $\mathrm{A}$, which also coincides with the location at which $\left(T_{u v}\right)_{\max }$ starts 

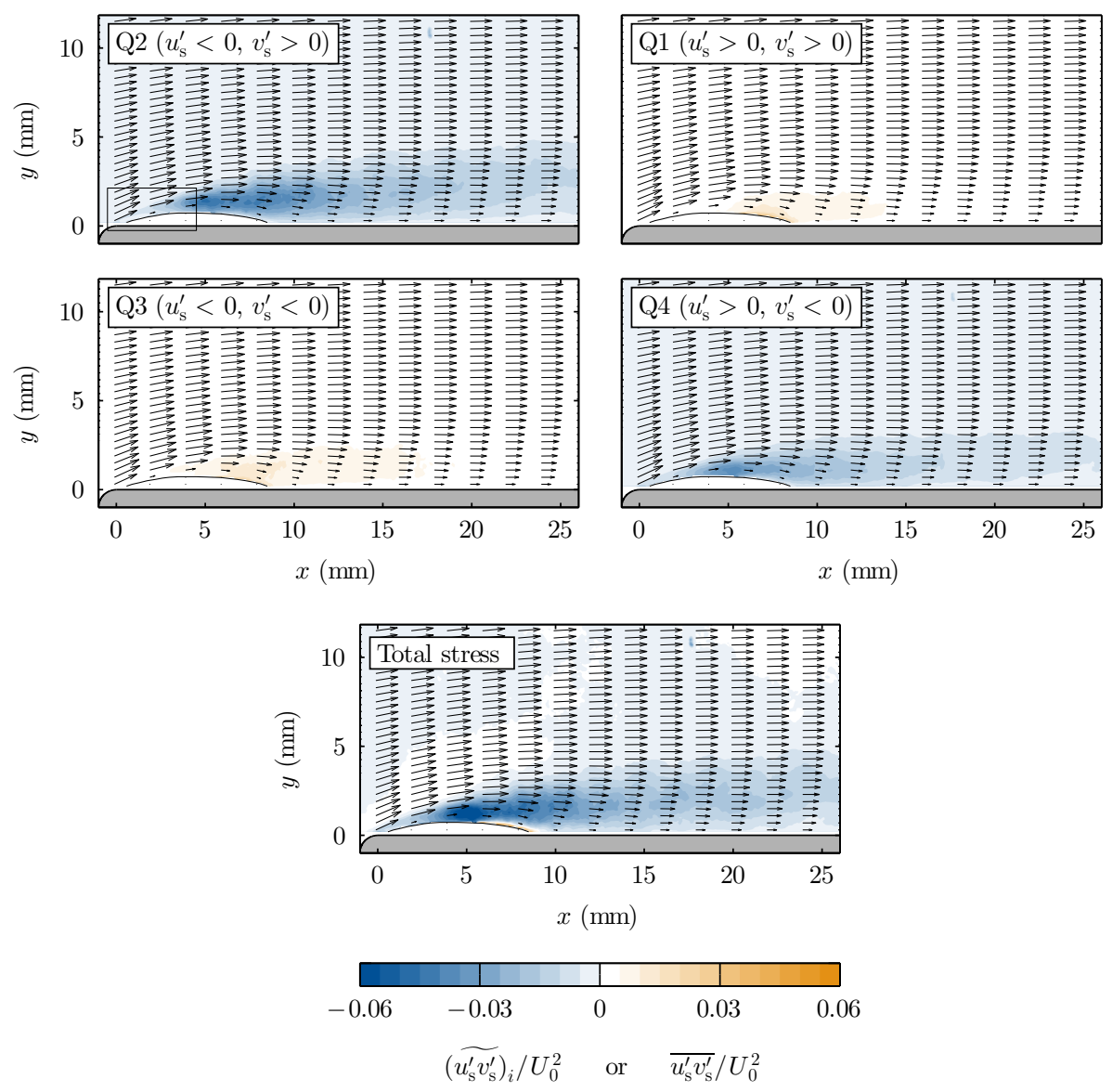

Figure 9: Contours of the stress contribution $\widetilde{\left(u_{\mathrm{s}}^{\prime} v_{\mathrm{s}}^{\prime}\right)_{i}} / U_{0}^{2}$ from each quadrant in case B (excluding the bubble region). Total Reynolds stress $\overline{u_{\mathrm{s}}^{\prime} v_{\mathrm{s}}^{\prime}} / U_{0}^{2}$ is shown at the base of the figure. Isolines of $\bar{u} / U_{0}=0$ are shown in black. Coarse profiles of the corresponding mean vector field have also been overlaid.

to rise considerably, the total stress increases due to large negative contributions from events in Q4 and Q2. These events broadly correspond to the movement of relatively highand low-speed fluid towards and away from the wall, respectively, although those in Q2 can still correspond physically to downflow. Case B is quite similar - contributions from Q2 and Q4 dominate the total stress in the latter part of the bubble-but particularly interesting is that the laminar part of the shear layer $(0<x<2.5 \mathrm{~mm})$ also receives significant contributions from these quadrants. We return to this point shortly.

Attention is first drawn to the vorticity plots in figures 10 and 11, which show roll-up of the shear layer in each case. Most experimental and computational studies of separation on blunt or round-nosed plates report the presence of these Kelvin-Helmholtz vortices; the high-resolution DNS of Lamballais et al. (2010) is one particularly lucid example and is qualitatively identical to what is seen here. Under elevated FST of the present magnitude, the only study of which we are aware is that of Langari \& Yang (2013), who suggest that whilst vortices fail to appear at the usual Kelvin-Helmholtz frequency, concentrated regions of vorticity $d o$ indeed emerge near the leading edge. We do not 

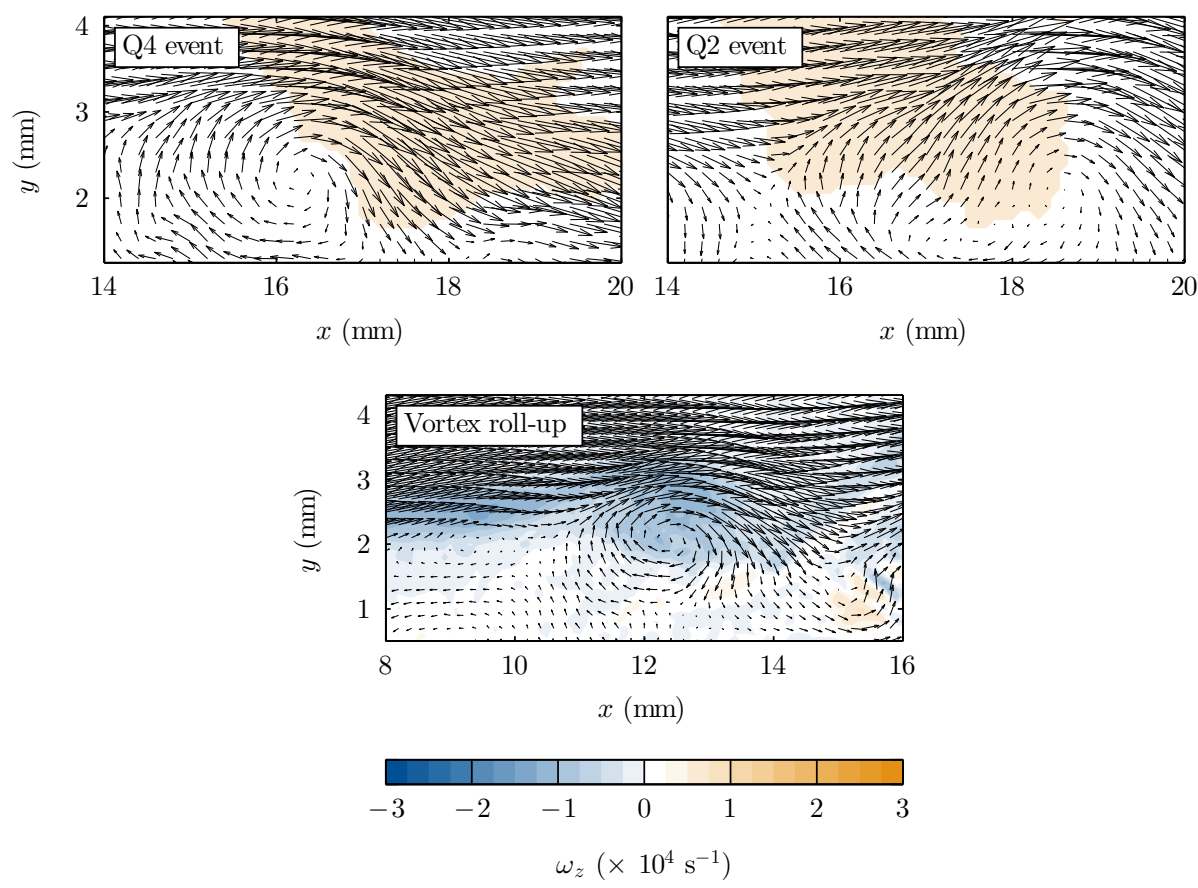

Figure 10: Fields of instantaneous absolute velocity in case A. The lower plot corresponds to the window on the left-hand contour in figure 6 and shows roll-up of the shear layer. The upper plots show typical Q4 and Q2 events from within the windows on the corresponding contours in figure 8 . Each has been contoured by a logical function that is true (orange) wherever that event is detected (in streamline or 's' coordinates).

here have the temporal resolution to comment upon shedding frequency but can confirm that distinct vortices, as shown in figure 11, are present in most of the instantaneous realisations of case B during the accelerated transition of the shear layer.

It was shown in figures 8 and 9 that in both cases the contributions to the total Reynolds stress from Q2 and Q4 were particularly strong over the latter half of the bubble. From a careful study of the individual vector fields in this region, it was subsequently found that the main transfer of momentum in Q4 results clearly from the type of event shown in the upper left-hand plot of figure 10, which shows fluid from upstream becoming entrained by a 'residual' KH vortex and diverted forcibly towards the wall at a speed greater than the mean. The corresponding region that actually generates Q4 stress (in streamline coordinates) has been contoured in orange. Note from figures 11 and 12 that the stress contribution is strongest at points that, on average, are: (i) far enough from the wall to experience entrainment only from the top side of the vortices; and (ii) far enough downstream for the vortices to have evolved to a sufficient strength. In fact, the mechanism is quite similar to the one suggested by Tafti \& Vanka (1991) in their numerical study and is presumably responsible for driving reattachment processes at the rear of the bubble. In the upper right-hand plot of figure 10, a typical Q2 event - an energetic upwelling of fluid - is shown. Whilst this particular event appears to have been induced by the rising side of a vortex, many in Q2 seem incoherent and do not.

For case B, it was also noted previously that the total stress in the laminar part of the shear layer-before $\left(T_{u v}\right)_{\max }$ reaches its peak - receives strong negative contributions 

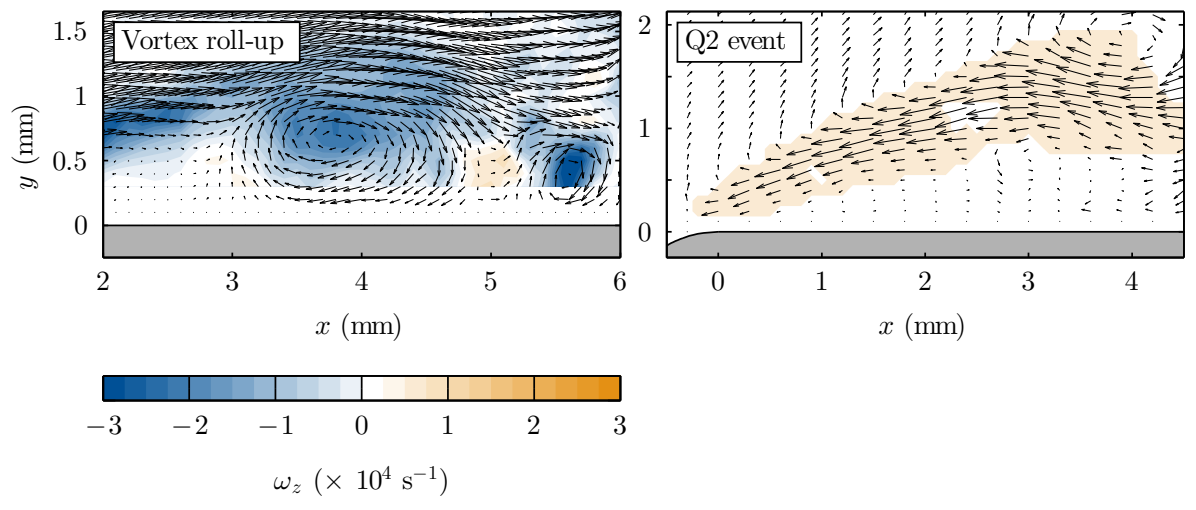

Figure 11: Fields of instantaneous velocity in case B. The left-hand plot corresponds to the window on the right-hand contour in figure 6 and shows roll-up of the shear layer in terms of the absolute velocity field $(u, v)$. The right-hand plot shows a typical Q2 event, as a Reynolds-decomposed fluctuation, from the window in the upper left-hand contour of figure 9 . The fluctuation has been contoured by a logical function, as in figure 10 .

from both Q2 and Q4. The right-hand plot in figure 11 shows one such event from Q2: a slender, yet highly coherent, negative $u_{\mathrm{s}}^{\prime}$ fluctuation (hence positive $v_{\mathrm{s}}^{\prime}$ ) that spans the full length of the laminar shear layer until the onset of roll-up. Similar positive fluctuations in Q4 have also been observed. In the side view shown here, these structures are reminiscent of the laminar streaks that are usually observed in pre-transitional boundary layers (Nolan \& Walsh 2012) and upstream of laminar separation bubbles (Taniguchi et al. 2012) under elevated FST. The vorticity isosurfaces presented by Langari \& Yang (2013) for a transitional free shear layer under 5.6 \% FST also appear to show corrugated fluctuations of a similar nature.

\subsection{The reattachment process}

Finally, we consider the reattachment process of the shear layer and the related question of 'flapping'. Plots (a) and (b) in figure 12 show the reverse-flow behaviour for two instantaneous realisations of case A (only this case is considered due to resolution limits near the wall). Each field has been contoured by isolines of $u=0$ into logical regions of forward $(u>0$, white) and reverse $(u<0$, blue) flow. Profiles of the instantaneous vector field have also been overlaid. The isoline is intended here as a device to reveal both the separation trajectory of the laminar part of the shear layer, with which it will move in tandem, and its unsteady reattachment behaviour downstream.

A common definition for the instantaneous reattachment point of a free shear layer is the last downstream location at which the skin friction is zero. This also corresponds to the last intersection between any isoline of $u=0$ and the wall. But in this flow, whilst there are times when the region of reverse flow at the leading edge constitutes a singular whole, as in (a), there are also times when it is fragmented into one or more isolated parts, as in (b). Some (or all) of these parts have no direct connection to the separating shear layer itself. By the usual definition, then, both of these bubbles would be regarded as having about the same instantaneous length, when in fact the main bubble of reverse flow at the leading edge differs in length by almost a factor of three. We therefore suggest that the downstream end of the isoline that extends unbroken from the separation point, termed here the 'separation isoline', be used to define the true location of instantaneous 

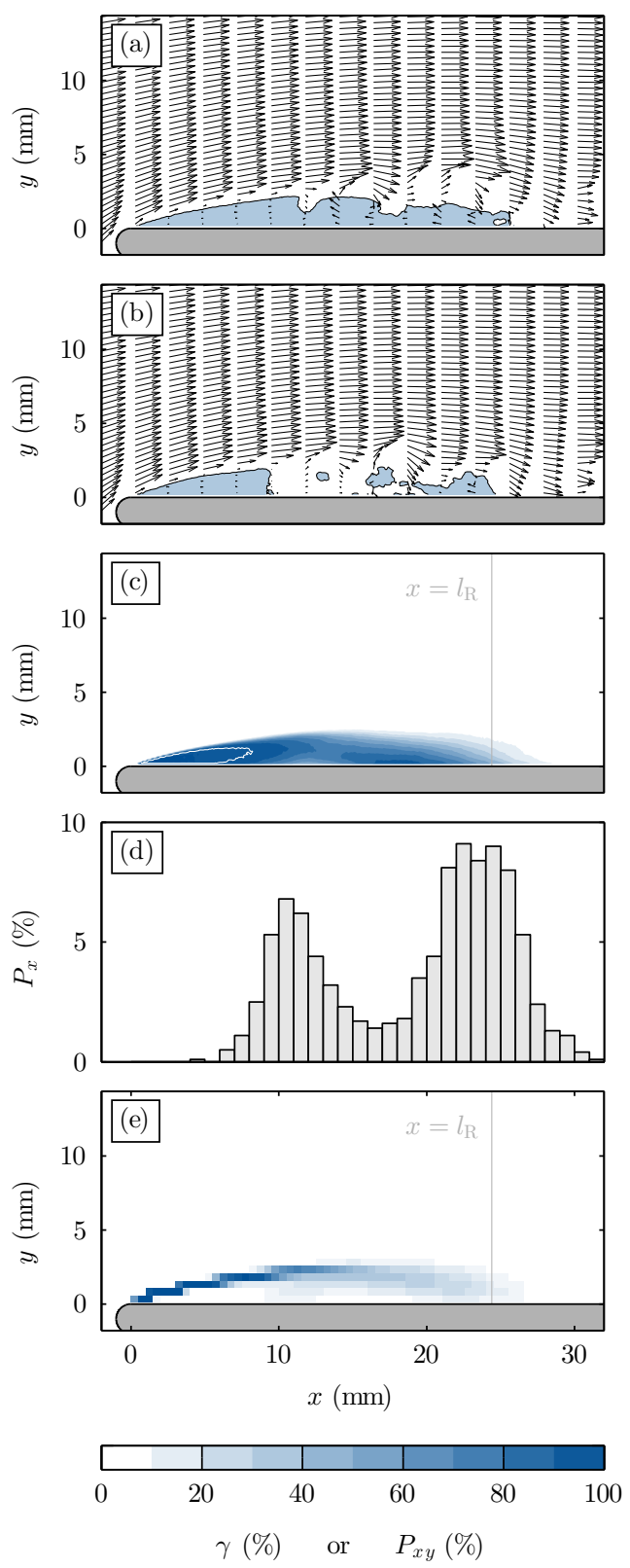

Figure 12: Reattachment and reverse-flow behaviour in case A. The instantaneous vector fields in (a) and (b) are contoured by isolines of $u=0$ and the regions they separate. The resulting distribution of the reverse-flow intermittency parameter $\gamma$ (the probability that $u<0$ at each point) is shown in (c), with a white line to denote $\gamma=99 \%$. In (d), a histogram of the reattachment point for the separation isoline is plotted in $1 \mathrm{~mm}$ bins. Last, in (e), the field has been divided into tiles of $0.25 \mathrm{~mm} \times 0.25 \mathrm{~mm}$, each of which is coloured according to the probability $P_{x y}$ that the separation isoline will lie within it during a realisation i.e. it is a joint PDF. 
reattachment (see also Castro 2005). Plot (c) shows the associated time-mean distribution of the reverse-flow intermittency parameter $\gamma$, which confirms that the separation isoline always bounds a region of reverse flow at the leading edge.

Plot (d) shows the probability distribution $P_{x}$ of this first reattachment point; it is distinctly bimodal. Both of the peaks are quite well defined, implying that the main bubble spends most of its time in either a 'short' or 'elongated' state. The upstream peak, corresponding to the former, coincides with the location of marked fluctuation growth $(x \simeq 10 \mathrm{~mm})$ in figures 6,7 and 8 , which suggests that this reattachment scenario may be linked to roll-up of (and shedding from) the laminar shear layer. The downstream peak, synonymous with elongated bubbles of the type shown in (a), is located very near the time-mean reattachment point. These bubbles must form by continuous reinjection from downstream, as described by Eaton \& Johnston (1982), either by means of (i) a very large structure, possibly a single vortex, or (ii) other smaller-scale structures. The former is unlikely in this case, however, because we have not observed any structures with a streamwise scale on the order of the distance between the peaks $\left(\simeq l_{R} / 2\right)$. Q4 vortex events (figure 10) would seem to provide one logical physical basis for the reattachment process that causes this peak, but examination of the vector fields is inconclusive. These events do not appear to be consistently associated with elongated bubbles in the present, stationary reference frame.

An even fuller picture of the main bubble is provided by a joint PDF of the probabilistic location $P_{x y}$ of the separation isoline between realisations. This is shown in (e). The diffusion of colour reveals horizontal and (slight) vertical movements that cause the reattachment point to jump along the wall in accordance with the two 'touchdown' peaks on the histogram. Such wide-scale motions are a well known feature of turbulent flows with low-frequency flapping (Eaton \& Johnston 1982; Yang \& Voke 2001). This fact, in combination with the absence (at present) of a definitive connection between regular KH shedding and the secondary peak, suggests that this flow may also be liable to a flapping motion involving the 'elongated' bubble, with the possibility of an associated global shedding mode (Castro 2005) - the maximum backflow velocity $\left(\simeq 0.3 U_{0}\right)$ and Reynolds number (790) are certainly high enough.

\section{Conclusions}

PIV measurements of a transitional separation-reattachment flow at the leading edge of a plate have been made for two levels of free-stream turbulence (FST) - background and elevated $(7 \%)$ - at relatively low Reynolds number. The flow was induced by means of a hinged flap at the trailing edge of the plate.

In each case, decomposition of the Reynolds stress revealed strong entrainment events in quadrants two (Q2) and four (Q4) towards the rear of the bubble. The latter were found physically to correspond to a forceful mechanism in which residual Kelvin-Helmholtz vortices drove fluid towards the wall. These vortices also seem to be an important part of the reattachment process, but their precise role is not yet fully clear. Further analysis showed that under elevated FST, the laminar part of the shear layer often experienced positive and negative fluctuations (versus the mean) that resemble the boundary-layer streaks usually seen in attached bypass transition. Subsequent transition of the shear layer was accelerated but also involved the roll-up of vortices. The resulting time-mean bubble was almost threefold shorter.

For the case of low FST, the instantaneous reattachment behaviour of the flow was studied using isolines of zero absolute streamwise velocity, or $u=0$. The bubble of reverse flow at the leading edge was found frequently to break up into two or more parts, thereby 
obscuring the reattachment process. We therefore suggest that the downstream end of the separation isoline (the one that is permanently anchored to the leading edge) be used to define the instantaneous reattachment point. A histogram of this point was found to be bimodal: the upstream peak corresponded approximately to the onset of roll-up in the shear layer, whereas the downstream peak was located near the time-mean reattachment point. The broad scale of the reattachment process - over half the time-mean bubble length - suggests that this shear layer may even exhibit a 'flapping' motion of the kind observed in fully turbulent reattaching flows.

Funding from the Irish Research Council (IRC) is very gratefully acknowledged. Bell Labs Ireland would also like to thank the Industrial Development Agency (IDA) Ireland.

\section{REFERENCES}

Bearman, P. W. \& Morel, T. 1983 Effect of free stream turbulence on the flow around bluff bodies. Progress in Aerospace Sciences 20 (2-3), 97-123.

Castro, I. P. 2005 The stability of laminar symmetric separated wakes. Journal of Fluid Mechanics 532, 389-411.

Castro, I. P. \& EPIK, E. 1998 Boundary layer development after a separated region. Journal of Fluid Mechanics 374, 91-116.

Castro, I. P. \& Haque, A. 1987 The structure of a turbulent shear layer bounding a separation region. Journal of Fluid Mechanics 179, 439-468.

Castro, I. P. \& Haque, A. 1988 The structure of a shear layer bounding a separation region. Part 2. Effects of free-stream turbulence. Journal of Fluid Mechanics 192, 577-595.

Chandrsuda, C. \& Bradshaw, P. 1981 Turbulence structure of a reattaching mixing layer. Journal of Fluid Mechanics 110, 171-194.

Charonko, J. J. \& Vlachos, P. P. 2013 Estimation of uncertainty bounds for individual particle image velocimetry measurements from cross-correlation peak ratio. Measurement Science and Technology 24 (6).

Cherry, N. J., Hillier, R. \& Latour, M. E. M. P. 1984 Unsteady measurements in a separated and reattaching flow. Journal of Fluid Mechanics 144, 13-46.

Djilali, N. \& Gartshore, I. S. 1991 Turbulent flow around a bluff rectangular plate. Part i: experimental investigation. ASME Journal of Fluids Engineering 113 (1), 51-59.

Dryden, H. L. 1943 A review of the statistical theory of turbulence. In Turbulence: Classical Papers on Statistical Theory (ed. S. K. Friedlander \& L. Topper), pp. 115-132. Interscience.

EAton, J. K \& Johnston, J. P. 1982 Low frequency unsteadiness of a reattaching turbulent shear layer. In Turbulent Shear Flows 3, pp. 162-170. Springer.

Fransson, J. H. M., Matsubara, M. \& Alfredsson, P. H. 2005 Transition induced by free-stream turbulence. Journal of Fluid Mechanics 527, 1-25.

Hernon, D., Walsh, E. J. \& McEligot, D. M. 2007 Experimental investigation into the routes to bypass transition and the shear-sheltering phenomenon. Journal of Fluid Mechanics 591, 461-479.

Hillier, R. \& Cherry, N. J. 1981 The effects of stream turbulence on separation bubbles. Journal of Wind Engineering and Industrial Aerodynamics 8 (1-2), 49-58.

Hudy, L. M., NAguib, A. M. \& Humphreys JR, W. M. 2003 Wall-pressure-array measurements beneath a separating/reattaching flow region. Physics of Fluids 15 (3), $706-717$.

Hunt, J. C. R. \& Durbin, P. A. 1999 Perturbed vortical layers and shear sheltering. Fluid Dynamics Research 24 (6), 375-404.

Hwang, K. S., Sung, H. J. \& Hyun, J. M. 2000 Visualizations of large-scale vortices in flow about a blunt-faced flat plate. Experiments in Fluids 29 (2), 198-201.

Kalter, M. \& Fernholz, H. H. 2001 The reduction and elimination of a closed separation region by free-stream turbulence. Journal of Fluid Mechanics 446, 271-308.

KANEvČE, G. \& OKA, S. 1973 Correcting hot-wire readings for influence of fluid temperature variations. DISA Information 15. DISA. 
KIYA, M. \& SASAKI, K. 1983 Structure of a turbulent separation bubble. Journal of Fluid Mechanics 137, 83-113.

KIYA, M. \& SASAKI, K. 1985 Structure of large-scale vortices and unsteady reverse flow in the reattaching zone of a turbulent separation bubble. Journal of Fluid Mechanics 154, 463-491.

Kurian, T. \& Fransson, J. H. M. 2009 Grid-generated turbulence revisited. Fluid Dynamics Research 41 (2).

Lamballais, E., Silvestrini, J. \& Laizet, S. 2010 Direct numerical simulation of flow separation behind a rounded leading edge: Study of curvature effects. International Journal of Heat and Fluid Flow 31 (3), 295-306.

Lane, J. C. \& Loenrke, R. I. 1980 Leading edge separation from a blunt plate at low Reynolds number. ASME Journal of Fluids Engineering 102 (4), 494-496.

LANGARI, M. \& YANG, Z. 2013 Numerical study of the primary instability in a separated boundary layer transition under elevated free-stream turbulence. Physics of Fluids 25 (7).

Li, X. \& DJILALI, N. 1995 On the scaling of separation bubbles. JSME International Journal Series B 38 (4), 541-548.

Lumley, J. L. 1970 Stochastic Tools in Turbulence. Dover.

Nolan, K. P. \& WALsh, E. J. 2012 Particle image velocimetry measurements of a transitional boundary layer under free stream turbulence. Journal of Fluid Mechanics 702, 215-238.

O’Neill, P. L., Nicolaides, D., Honnery, D. \& Soria, J. 2004 Autocorrelation functions and the determination of integral length with reference to experimental and numerical data. In 15th Australasian Fluid Mechanics Conference, pp. 13-17.

Ota, T., Asano, Y. \& Okawa, J. 1981 Reattachment length and transition of the separated flow over blunt flat plates. Bulletin of the JSME 24 (192), 941-947.

RoAch, P. \& Brierley, D. H. 1992 The influence of a turbulent free-stream on zero pressure gradient transitional boundary layer development part I: test cases T3A and T3B. In Numerical Simulation of Unsteady Flows and Transition to Turbulence, pp. 319-347. Cambridge University Press.

RoACH, P. E. 1987 The generation of nearly isotropic turbulence by means of grids. International Journal of Heat and Fluid Flow 8 (2), 82-92.

Ruderich, R. \& Fernholz, H. H. 1986 Experimental investigation of a turbulent shear flow with separation, reverse flow, and reattachment. Journal of Fluid Mechanics 163, 283322.

SASAKI, K. \& KIYA, M. 1985 Effect of free-stream turbulence on turbulent properties of a separation-reattachment flow. Bulletin of the JSME 28 (238), 610-616.

SASAKI, K. \& KIYA, M. 1991 Three-dimensional vortex structure in a leading-edge separation bubble at moderate Reynolds numbers. ASME Journal of Fluids Engineering 113 (3), 405-410.

ScArAno, F. 2002 Iterative image deformation methods in PIV. Measurement Science and Technology 13 (1), R1-R19.

Stevenson, J. P. J., Walsh, E. J., Nolan, K. P. \& Davies, M. R. D. 2014 Separation \& free-stream turbulence: implications for surface aerodynamics \& heat transfer. Journal of Physics: Conference Series $\mathbf{5 2 5}$ (1).

TAFti, D. K. \& VANKA, S. P. 1991 A numerical study of flow separation and reattachment on a blunt plate. Physics of Fluids A 3 (7), 1749-1759.

TAni, I. 1964 Low-speed flows involving bubble separations. Progress in Aerospace Sciences 5, 70-103.

Taniguchi, H., Sakai, H. \& Funazaki, K. 2012 Effects of freestream turbulence on bypass transition of separated boundary layer on low-pressure turbine airfoils. Journal of Thermal Science 21 (3), 230-235.

YANG, Z. \& ABDAlla, A. E. 2009 Effects of free-stream turbulence on a transitional separatedreattached flow over a flat plate with a sharp leading edge. International Journal of Heat and Fluid Flow 30 (5), 1026-1035.

YANG, Z. \& ABDAlla, I. E. 2005 Effects of free-stream turbulence on large-scale coherent structures of separated boundary layer transition. International Journal for Numerical Methods in Fluids 49 (3), 331-348. 
YANG, Z. \& ABDAlla, I. E. 2008 On coherent structures in a separated/reattached flow. WSEAS Transactions on Fluid Mechanics 3, 143-153.

YANG, Z. \& VOKE, P. R. 2001 Large-eddy simulation of boundary-layer separation and transition at a change of surface curvature. Journal of Fluid Mechanics 439, 305-333. 